\title{
Time to positivity of neonatal blood cultures
}

\author{
Y Kumar, M Qunibi, T J Neal, C W Yoxall
}

\begin{abstract}
Aim-To determine how long it takes neonatal blood cultures to become positive. Methods-Data were collected retrospectively on 451 positive blood cultures from babies on a tertiary neonatal unit between January 1997 and December 1998. During the study period, the laboratory used the BacT/Alert microbial detection system. Results-Complete information was available on 416 blood cultures. Twelve became positive after 72 hours, none of which were considered to be clinically significant. Of the 404 remaining cultures, $86 \%$ were positive at 36 hours, $96 \%$ at 48 hours, and $98.5 \%$ by 60 hours. If definite bacterial pathogens are considered alone, the time to positivity was $90 \%$ by 36 hours, $93 \%$ by 48 hours, and $98 \%$ by 60 hours. If definite and possible bacterial pathogens are considered (coagulase negative staphylococci taken as possible bacterial pathogens), the time to positivity was $89 \%$ at 36 hours and $97 \%$ at 48 hours. The negative predictive value, for isolation of any organism before 72 hours, of a negative blood culture was $97 \%$ at 36 hours and $99 \%$ at 48 hours. The negative predictive value for the isolation of definite bacterial pathogens only was $99.7 \%$ at 36 hours and $99.8 \%$ at 48 hours.

Conclusions-A period of 36 hours is enough to rule out sepsis in the asymptomatic neonate, and a three day incubation period is sufficient to detect all clinically important infections using the BacT/Alert microbial detection system.

(Arch Dis Child Fetal Neonatal Ed 2001;85:F182-F186)
\end{abstract}

Keywords: blood cultures; positivity; sepsis; infection

Infection is an important cause of mortality and morbidity in the neonatal unit. The early symptoms of sepsis are non-specific, and the outlook is considered to be worst in babies in whom antibiotics are started late. ${ }^{1}$ Antibiotics are therefore generally given to all babies with clinical or laboratory indicators of infection and babies at high risk of early onset sepsis. A large number of babies who are evaluated for sepsis do not have proven infection. ${ }^{23}$ This means that most of the antibiotics given to babies on the neonatal unit are given to babies without infection. Inappropriate use of antibiotics has been implicated in the development of multiresistant bacteria in hospitals. ${ }^{4}$

To reduce the antibiotic pressure on the neonatal unit, it is common practice to stop antibiotic treatment if the blood culture is negative at $48-72$ hours and the baby does not have any clinical or laboratory indicators of infection. This practice is based on four studies of the time to positivity for neonatal blood cultures. ${ }^{2356}$ All of these studies are based on relatively small numbers of positive blood cultures (less than 225 in each case), and three of the studies took place before $1990 .^{235}$ Two of the studies used laboratory techniques that have been superseded and did not consider coagulase negative staphylococci as pathogens, ${ }^{25}$ although these are now the most common organisms isolated in intensive care nurseries. ${ }^{7}$ Recently published studies suggest that antibiotic courses may be safely reduced to 24-36 hours in asymptomatic term babies. ${ }^{8}$

The aim of this study was to measure the time taken for bacteria to be detected in blood cultures taken from babies on the neonatal unit using the BacT/Alert microbial detection system (Organon Teknika Corporation, Durham, North Carolina, USA). The purpose was to investigate whether antibiotic treatment could be discontinued before 48 hours in babies with negative blood cultures, so reducing antibiotic pressure on the neonatal unit.

\section{Methods}

A two year (January 1997 to December 1998) retrospective study of babies was completed on the neonatal unit at Liverpool Women's Hospital, a tertiary level III neonatal unit. A total of 451 positive blood cultures were identified from the microbiology database maintained at Royal Liverpool University Hospital, which provides the microbiology services for Liverpool Women's Hospital. The BacT/Alert microbial detection system was in use at the Royal Liverpool University Hospital during the study period.

Blood ( $0.5 \mathrm{ml}$ or more) was collected by venepuncture, after the skin had been cleaned with $70 \%$ alcohol, and inoculated into a BacT/ Alert aerobic blood culture bottle. The bottle was then incubated immediately at $37^{\circ} \mathrm{C}$. The time to positivity of a blood culture was taken as the time from inoculation of the blood into the culture bottle to the time at which the BacT/Alert machine signalled a positive result.

The BacT/Alert bottle contains $20 \mathrm{ml}$ brain/ heart infusion broth, with amino acid and carbohydrate substrates, and $0.02 \%$ sodium polyanetholesulphonate as an anticoagulant. ${ }^{10}$ The substrates in the aerobic culture bottle are in an atmosphere of carbon dioxide at subatmospheric pressure and are designed for the growth of common aerobic, microaerophilic, and fastidious bacteria and common yeasts. The aerobic blood culture bottle alone is used on the unit. The temperature is maintained at $35-37^{\circ} \mathrm{C}$. Each culture bottle contains a colorimetric carbon dioxide sensor to measure 
Table 1 Classification of organisms isolated in 416 positive blood cultures

\begin{tabular}{|c|c|c|c|c|c|c|c|}
\hline Definite pathogens & & Possible pathogens & & Fungi & & Contaminants & \\
\hline$S$ aureus & 4 & CoNS & 266 & $C$ albicans & 25 & Diphtheroids & 12 \\
\hline$S$ marcescens & 8 & Two or more organisms & 55 & $C$ parapsilosis & 7 & Bacillus sp & 1 \\
\hline$P$ aeruginosa & 8 & E faecalis & 1 & Yeasts & 1 & Micrococcus & 4 \\
\hline Kpneumoniae & 2 & $S$ mitis & 1 & & & & \\
\hline Koxytoca & 1 & & & & & & \\
\hline GBS & 5 & & & & & & \\
\hline E coli & 12 & & & & & & \\
\hline Enterobacter $\mathrm{sp}$ & 1 & & & & & & \\
\hline E cloacae & 2 & & & & & & \\
\hline Total & 43 & Total & 323 & Total & 33 & Total & 17 \\
\hline
\end{tabular}

microbial growth. Because growth of microorganisms produces carbon dioxide, the colour of the gas permeable sensor changes from green to yellow. The BacT/Alert system tests for carbon dioxide production every $10 \mathrm{~min}$ utes, and data points are plotted. In this system, each bottle is compared with itself, rather than using a predetermined threshold value as other systems have used. As the amount of carbon dioxide dissolved in the culture medium rises, a light emitting diode reflects more light, indicating to the computer that the blood culture is positive. This immediately sets off an alarm, and the time that the blood culture became positive is recorded in the data management system. No routine subculturing is necessary. This is a completely automated system that continuously agitates and monitors the specimen during incubation. The bottles are incubated for seven days.

Basic, clinical, and laboratory data were collected on all the babies for each positive blood culture episode. The organism isolated and the timing of the positive signal were obtained from the microbiology database. The remaining information was obtained from the case records.

The positive blood cultures were classified on the basis of the organism isolated as bacterial or fungal. Bacteria were further subdivided into definite pathogens, possible pathogens, and contaminants. Definite pathogens were defined as organisms known to cause disease in the newborn-for example, Group B streptococcus (GBS), Staphylococcus aureus, Group G streptococcus, Escherichia coli, Klebsiella pneumoniae, Enterobacter cloacae, and Citrobacter diversus. Possible pathogens were defined as organisms known to cause disease under special circumstances - for example, immunosupressed host, presence of an indwelling catheter. This group contained coagulase negative staphylococci, $\alpha$-haemolytic streptococci, nonhaemolytic streptococci, and anaerobes. Blood cultures containing two or more organisms were considered to be possible pathogens if one of them was a definite or possible pathogen. ${ }^{11}{ }^{12}$ Contaminants were defined as organisms that rarely cause disease in the newborn-for example, Corynebacterium species (diphtheroids), Propionibacterium species, and nonviable organisms.

Microsoft Office 97 package (Access and Excel) and "Arcus for Windows" package were used for statistical analysis. Mann-Whitney U tests were used to compare times to positivity for the various groups. Ethical approval for the study was obtained from the Paediatric Research Ethics Committee, Alder Hey Children's Hospital.

\section{Results}

During the two year study period, there were 12117 live births in Liverpool Women's Hospital. There were 1466 admissions to the neonatal unit, of whom 210 were postnatal transfers from other hospitals. Of the 2268 blood cultures obtained from the babies, 451 $(19.9 \%)$ were positive from 215 babies with a median (5th and 95th centile) number of blood cultures from each baby of 1 ( 1 and 5 ); $68 \%$ of the blood cultures were from male babies. The median (5th and 95th centile) gestation of the babies with positive blood cultures was 28 (24 and 36) weeks. The median (5th and 95th centile) birth weight was 1110 (622 and 2809) g. The median (5th and 95th centile) age at blood culture collection was 14 (0 and 97) days.

Complete information was available on 416 of the $451(92.2 \%)$ positive blood cultures. Thirty of the $416(7.2 \%)$ blood samples were drawn within 48 hours of birth. Table 1 shows all the organisms isolated in the blood cultures.

Table 2 gives the time to positivity of all the blood cultures. Twelve of the $416(2.9 \%)$ blood cultures that became positive after 72 hours were not considered by the attending clinicians to be indicative of true infections. All 12 babies were asymptomatic, and antibiotic treatment had already been discontinued by the time the positive blood culture result had become available.

Of the 16 blood cultures that became positive between 48 and 72 hours, eight were possible pathogens, four were fungi, two were $E$ coli, one was a Serratia marcescens, and one a contaminant. Of the 41 blood cultures that became positive between 36 and 48 hours, 27 were possible pathogens, seven were fungi, six were contaminants, and one was a $S$ marcescens.

The median (5th and 95th centile) time to positivity of the 43 organisms in the definite bacterial pathogen group was 17.9 (9.5 and 49.6) hours. Two of the three babies with definite bacterial pathogens isolated in blood cultures after 48 hours had $E$ coli septicaemia and were receiving antibiotics at the time of collection of the blood samples. Both of these 
Table 2 Duration of time for blood cultures to become positive

\begin{tabular}{|c|c|c|c|c|c|c|c|}
\hline & 0-12 hours & 12-24 hours & 24-36 hours & 36-48 hours & 48-60 hours & 60-72 hours & $>72$ hours \\
\hline Total positives & $10(2.4)$ & $183(44)$ & $154(37)$ & $41(9.9)$ & $10(2.4)$ & $6(1.4)$ & $12(2.9)$ \\
\hline Definite bacterial pathogens & $5(11.6)$ & $28(65.1)$ & $6(14)$ & $1(2.3)$ & $2(4.3)$ & $1(2.3)$ & 0 \\
\hline CoNS & $3(1.1)$ & $118(44.4)$ & $110(41.3)$ & $25(9.4)$ & $3(1.2)$ & $4(1.5)$ & $3(1.1)$ \\
\hline Possible bacterial pathogens (including CoNS) & $4(1.25)$ & $153(47.4)$ & $127(39.3)$ & $27(8.3)$ & $4(1.25)$ & $4(1.25)$ & $4(1.25)$ \\
\hline Contaminants & 0 & 0 & $2(11.8)$ & $6(35.3)$ & $1(5.9)$ & 0 & $8(47)$ \\
\hline Fungi & $1(3)$ & $2(6.1)$ & $19(57.6)$ & $7(21.2)$ & $3(9.1)$ & $1(3)$ & 0 \\
\hline
\end{tabular}

Values in parentheses are percentages.

CoNS, Coagulase negative staphylococci.

babies showed clear evidence of necrotising enterocolitis: one had undergone laparotomy, and an abdominal drain had been inserted in the other. There were two babies with $S$ marcescens isolated from blood cultures after 36 hours, one at 37 hours and the other at 51 hours. Neither of these babies were receiving antibiotics at the time of blood collection. The baby with $S$ marcescens isolated from blood culture at 51 hours was considered to have clinical evidence of sepsis, with a decision to continue the antibiotics when the blood cultures were negative at 48 hours.

The time to positivity for culture of definite pathogens in babies who were receiving antibiotics before the blood samples were taken was compared with those who were not. The median times to positivity were 21.4 and 17.7 hours respectively. The difference was not statistically significant $(\mathrm{p}=0.6)$.

Coagulase negative staphylococci accounted for $63.9 \%(266 / 416)$ of the total isolates. The times to positivity of the coagulase negative staphylococci isolates were compared with those of the definite bacterial pathogens. The median times to positivity were 24.6 and 17.9 hours respectively. The difference of 6.7 hours was statistically significant $(p=0.001)$.

The median (5th and 95th centile) time to positivity of the $33(7.9 \%)$ fungal isolates was 32.6 (15.3 and 55.8) hours. All four babies whose blood cultures were positive for fungi after 48 hours were receiving amphotericin at the time of blood collection. However, there

Table 3 Comparison of culture status at 36 and 48 hours in relation to eventual status

$\begin{array}{lll}\text { (a) All positive cultures (those positive after } 72 \text { hours classified as negative) } \\ & \text { Positive at } 36 \text { hours } & \text { Negative at } 36 \text { hours } \\ \text { Positive } & 347 & 57 \\ \text { Negative } & 0 & 1829 \\ & & \\ & \text { Positive at } 48 \text { hours } & \text { Negative at } 48 \text { hours } \\ \text { Positive } & 388 & 16 \\ \text { Negative } & 0 & 1829\end{array}$

(b) All definite bacterial pathogens and possible bacterial pathogens (contaminants reclassified as negative; fungal cultures removed from analysis)

$\begin{array}{lll} & \text { Positive at } 36 \text { hours } & \text { Negative at } 36 \text { hours } \\ \text { Positive } & 323 & 40 \\ \text { Negative } & 0 & 1838 \\ & & \\ & \text { Positive at } 48 \text { hours } & \text { Negative at } 48 \text { hours } \\ \text { Positive } & 351 & 12 \\ \text { Negative } & 0 & 1838\end{array}$

(c) Definite bacterial pathogens only (contaminants reclassified as negative; fungal cultures and possible bacterial pathogens removed from analysis)

$\begin{array}{lll} & \text { Positive at } 36 \text { hours } & \text { Negative at } 36 \text { hours } \\ \text { Positive } & 39 & 4 \\ \text { Negative } & 0 & 1838 \\ & \text { Positive at } 48 \text { hours } & \text { Negative at } 48 \text { hours } \\ \text { Positive } & 40 & 3 \\ \text { Negative } & 0 & 1838\end{array}$

was no significant effect of antifungal treatment before blood collection on the time to positivity of the fungal isolates. The median (range) time to positivity was $35.8(28.4-60.8)$ hours in the 10 blood cultures obtained from babies receiving treatment and $31.6(14.6-41.5)$ hours in the 23 blood cultures obtained from babies not receiving treatment $(\mathrm{p}=0.06)$.

Assuming that all significant bacteraemia or fungaemia will yield a positive culture by 72 hours, if all positive cultures were considered, the sensitivity for detection of bacteraemia or fungaemia after 36 hours and 48 hours incubation was $86 \%$ and $96 \%$ respectively (table $3 a$ ).

If only definite and possible bacterial pathogens are considered - that is, contaminants are reclassified as negative, and fungal positive cultures are removed from the analysis-the sensitivity for significant bacteraemia at 36 and 48 hours incubation was $88.9 \%$ and $96.6 \%$ respectively. The negative predictive value for bacteraemia of a negative blood culture $(95 \%$ confidence interval) was $97.9 \% \quad(97.2$ to $98.5 \%$ ) and $99.4 \%(99.0$ to $99.7 \%)$ at 36 and 48 hours respectively (table $3 \mathrm{~b}$ ).

When isolates of definite bacterial pathogens only were considered - that is, contaminants reclassified as negative, and fungal and possible bacterial pathogens excluded from the analysis - the sensitivity at 36 and 48 hours was $90.1 \%$ and $93 \%$ respectively. The negative predictive value (95\% confidence interval) at 36 and 48 hours for definite bacterial pathogens was $99.7 \%$ (99.5 to $99.9 \%)$ and $99.8 \%$ (99.7\% to $100 \%$ ) respectively (table $3 \mathrm{c}$ ).

\section{Discussion}

This is the second neonatal study to investigate the time to positivity using a single blood culture bottle system. ${ }^{8}$ The use of the single paediatric BacT/Alert aerobic bottle for growth of both aerobic and anaerobic organisms has been validated..$^{13} 14$

The proportion of the total number of blood cultures that were positive was about $20 \%$, whereas in all previous studies it was less than $10 \% .^{23} \mathrm{~A}$ possible reason may be a higher threshold for performing a septic screen on our neonatal unit. The spectrum of organisms isolated from positive blood cultures in our study is different from previous studies. ${ }^{23568}$ Some $10 \%$ of the positive blood cultures contained definite bacterial pathogens in our study whereas 30\% were definite bacterial pathogens in similarly analysed studies. ${ }^{3}{ }^{8}$ Some $77.6 \%$ of the total isolates were classified as possible bacterial pathogens in this study whereas only $38 \%$ were possible bacterial pathogens in the 
1989 study of Kurlat et $a l^{3}$ and $51 \%$ in the recent study of Garcia-Prats et $a l^{8}{ }^{8}$ This difference is due to an increase in the proportion of coagulase negative staphylococci isolates and is a reflection of the differences in the study populations. Changes in neonatal intensive care over the past 10 years have led to more extremely low birthweight, extremely preterm babies surviving for longer periods. Most of the previous studies did not report on the group of blood cultures with more than one organism isolated from each blood culture bottle. ${ }^{23} 6$ This group contributed $13.2 \%$ of the total isolates in the study. Fungi constituted about $8 \%$ of the total organisms, a finding similar to other studies. ${ }^{368}$

The time to positivity of blood cultures in the study was shorter than in earlier studies, ${ }^{2356}$ but similar to the findings of recent studies that used modern non-radiometric blood culture incubation techniques. ${ }^{89}$ This may be because of advances in laboratory methodology such as completely automated microbial detection systems. Another factor may be the shorter gestation of the babies in our study than in the earlier work. Preterm babies may have a higher bacterial load per $\mathrm{ml}$ of blood compared with term neonates and infants, resulting in quicker growth of organisms. Neonates with bacteraemia have been reported to have a much higher concentration of organisms per $\mathrm{ml}$ of blood than adults. ${ }^{15}$ Another explanation may be that the culture bottles may have been inoculated with a larger volume of blood in this study than in other studies. However, we did not look at the relation between volume of blood inoculated into the culture bottles and time to positivity.

For the four babies in whom blood cultures became positive after 36 hours for definite bacterial pathogens, a negative blood culture result at 36 or 48 hours would not have led to the discontinuation of antibiotic treatment in two because of their recent surgery and clinical status. The third may have had the antibiotic treatment discontinued at 36 hours, only to be restarted at 37 hours. This would not have led to any antibiotic doses being missed. It is unlikely that the last baby would have had the antibiotic treatment inappropriately stopped by a negative result at 36 hours, as he was still considered to be clinically septic and the treatment was continued with a negative result at 48 hours.

If a blood culture was negative at 36 hours, it was $98 \%$ probable to remain negative for definite or possible bacterial pathogens. The diagnostic yield was not significantly improved by prolonging the incubation to 48 hours. For definite bacterial pathogens alone, the probability of a blood culture negative at 36 hours remaining negative at 72 hours was $99.8 \%$, and this was not improved by increasing the incubation period to 48 hours.

It can be concluded that a 36 hour observation period is enough to rule out sepsis in the asymptomatic baby. If the baby remains symptomatic of a possible septicaemia at 36 hours after collection of blood, repeat blood cultures should be performed, and consideration should be given to starting a second line antibiotic regimen. Blood culture results should not be considered as a gold standard for sepsis and it may of course be necessary for some symptomatic babies to be treated as infected even though blood cultures remain negative. The study suggests that a three day observation period is sufficient to detect all clinically important blood culture isolates, thereby making the incubation of neonatal blood culture beyond 72 hours unnecessary.

In this study, $100 \%$ of the cultures from which fungi were isolated became positive by 48 hours if the babies had not received prior specific antifungal treatment. However, the number of babies in this part of the study was small.

The study was unable to show any effect of prior specific antimicrobial treatment on time to positivity of various groups of bacterial pathogens and fungi. Some of the babies in the study may have been exposed to maternally administered antibiotics antenatally or during labour. As administration of antibiotics to the babies themselves before blood collection had no effect on the time to positivity, it seems unlikely that maternally administered antibiotics would have had a significant effect.

There are enormous potential benefits from the application of the study findings provided that there is good communication between the microbiology laboratory and the neonatal unit. In our unit, to maximise the clinical impact of the automated system, there is a computer link between the neonatal unit and the microbiology laboratory. As soon as a positive result is signalled, it is instantly available on the neonatal unit. An estimate, based on present practice, shows that there would be a reduction in total antibiotic use on the neonatal unit of $15 \%$ if 36 hours instead of 48 hours was taken as the time limit for stopping antibiotic treatment when blood cultures are negative. The decreased use of antibiotics may reduce the emergence of antibiotic resistant strains of bacteria in the long term. ${ }^{16}$ There may be cost savings through decreased length of stay of babies in hospital, decreased use of materials such as intravenous cannulae, decreased use of antibiotics, and decreased length of incubation period for blood cultures. The workload of the neonatal staff may be reduced, particularly that of the junior doctors and nursing staff. If antibiotic treatment was stopped in asymptomatic babies with negative blood cultures at 36 hours, the babies would suffer less pain because of the use of fewer invasive procedures such as insertion of intravenous cannulae for antibiotic administration and blood tests such as antibiotic assays. Early discontinuation of antibiotics may result in earlier discharge of some of the babies, helping to improve bonding between parents and children.

This research was undertaken by YK for a MMedSc in Child Health, Unversity of Leeds, UK

\footnotetext{
1 Isaacs D, Moxon ER. Neonatal infections [preface]. Oxford: Butterworth-Heinemann, 1991.

2 Rowley AH, Wald ER. Incubation period necessary to detect bacteremia in neonates. Pediatr Infect Dis $f$ 1986;5:590-1.
} 
3 Kurlat I, Stoll BJ, McGowan JE. Time to positivity for detection of bacteremia in neonates. 7 Clin Microbiol

4 Crosson FJ, Moxon ER. Factors influencing kanamycin resistance in gram-negative enteric neonatal sepsis. Pediatrics 1978;61:478.

Pichichero ME, Todd JK. Detection of neonatal bacteremia f Pediatr 1979;94:958-60.

6 Hurst MK, Yoder BA. Detection of bacteremia in young infants: Is 48 hours adequate? Pediatr Infect Dis $\mathcal{F}$ 1995; 14:711-13.

7 Isaacs D. Rationing antibiotic use in neonatal units. Arch Dis Child Fetal Neonatal Ed 2000;82:F1-2.

8 Garcia-Prats JA, Cooper TR, Schneider VF, et al. Rapid detection of microorganisms in blood cultures of newborn infants utilizing an automated blood culture system. Pediatrics 2000;105:523-7.

9 Pauli I Jr, Shekhawat P, Kehl S, Sasidharan P. Early detection of bacteremia in the neonatal intensive care unit using the new BACTEC system. F Perinatol 1999;19:12731 .
10 Wilson $M$, Weinstein M, Reller L. Automated blood culture systems. Clin Lab Med 1994;14:149-69.

1 Isaacs D, Moxon ER. Antibiotic policies. In: Neonatal infections. Oxford: Butterworth-Heinemann, 1991.

12 Faix RG, Kovarik SM. Polymicrobial sepsis among intensive care nursery infants. F Perinatol 1989;9:131-6.

13 Krisher KK, Whyburn DR, Koepnick F. Comparison of the BacT/Alert Pediatric Blood Culture System, Pedi-BacT, with conventional culture using the 20-milliliter BectonDickinson Supplemented Peptone Broth Tube. F Clin Microbiol 1993;31:793-7.

14 Pickett DA, Wewlch DF. Evaluation of the Automated BacT/AlerT System for pediatric blood culturing. Clinical Microbiology and Infectious Diseases 1995;103:320-3.

15 Phillips SE, Bradley JS. Bacteremia detected by lysis direct plating in a neonatal intensive care unit. F Clin Microbiol 1990;28:1-4.

16 Lacey RW. Evolution of organisms and antibiotic resistance. Lancet 1984;ii: $1022-5$.

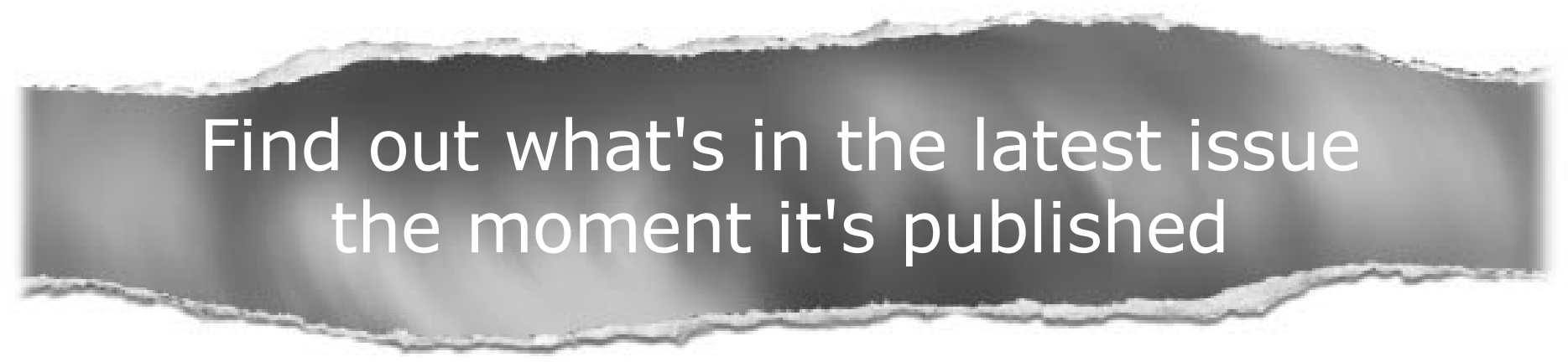

Email Alerts

Sign up to receive the table of contents by email every month. You can select from three alerts:

Table of Contents (full), TOC Awareness (notice only); Archives of Disease in Childhood related announcements.

www.archdischild.com 